\title{
A New Angle on Classifying Coronary Artery Bifurcation Stenoses
}

\author{
- The DINO System -
}

Andre Manica, MD; Julio Marchini, MD, PhD; Kevin Croce, MD, PhD

$\mathbf{O}$ ver the past 3 decades continued technological innovation has improved the safety and efficacy of percutaneous coronary intervention (PCI). Advances in guidewire, balloon, bare metal stent, and drug-eluting stent technologies have resulted in marked improvement in short term procedural success and in clinical cardiovascular outcomes for PCI patients. Despite the excellent results of PCI in simple lesions, significant challenges remain in the treatment of bifurcation stenoses, which currently account for approximately $20 \%$ of coronary interventions. ${ }^{1}$ Compared to singlevessel procedures, procedural success and clinical outcomes are significantly worse following bifurcation $\mathrm{PCI},{ }^{2}$ and interventional cardiologists are challenged by suboptimal treatment strategies for bifurcation disease.

\section{Article p 299}

Several different PCI techniques have been developed to treat coronary artery bifurcation stenoses. Culote, crush, $\mathrm{T}$-stent, and kissing-stent, are the predominant bifurcation PCI techniques; ${ }^{3}$ however, randomized studies have consistently demonstrated that a single-stent provisional strategy of stenting just the main branch (MB) has the best clinical outcomes compared to 2 -stent techniques. ${ }^{4-8}$ Indeed, in studies comparing provisional MB stenting to a planned 2-stent strategy of stenting both branches, the 2-stent approach showed no major benefit in follow up angiographic stenosis or major adverse cardiovascular events, and was associated with significant periprocedural increases in myocardial necrosis biomarkers, contrast utilization, and fluoroscopy time. ${ }^{4}$ Despite best efforts to utilize a provisional strategy, modern investigations of drug-eluting stent bifurcation stenting demonstrate that bifurcations treated with a provisional strategy will frequently require balloon dilation and bail-out implantation of a second stent in the side branch (SB). ${ }^{3}$ Importantly, the rates of bail-out SB stenting following a single-stent MB provisional strategy vary based on trial definitions for when a SB should be treated. In the CACTUS study, SB stenting was recommended following provisional single-stent MB PCI when the SB stenosis was greater than $50 \%{ }^{8}$ In CACTUS, $31 \%$ of the provisional single-stent-treated bifurcations required SB stenting. The Nordic bifurcation study, recommended balloon dilation only if the SB had less than Thrombolysis in
Myocardial Infarction (TIMI)-3 flow, and recommended SB stenting only if the SB had TIMI-0 flow following balloon dilation. ${ }^{4}$ Because of the higher tolerance for SB stenosis in the Nordic study, $32 \%$ of provisional single-stent-treated bifurcations were treated with SB balloon dilation, and only $4.3 \%$ were treated with bail-out SB stenting.

In order to optimize treatment strategies for bifurcation stenosis, and to facilitate research on the efficacy of various bifurcation PCI techniques, several bifurcation classification strategies have been developed. ${ }^{9-12}$ These classification strategies categorize bifurcation stenoses based on anatomic parameters that include stenotic involvement of $\mathrm{MB}$ and/or SB, size of the SB, and angulation of the SB. Experientially, these anatomic features strongly influence acute PCI success, measured by angiographic patency, residual stenosis of the MB or $\mathrm{SB}$, and periprocedural myocardial infarction. Less is known about the impact of bifurcation stenosis anatomy or angle on clinical outcomes; however, it is certainly possible that the extent of MB or SB disease and bifurcation angle could impact target lesion revascularization or stent thrombosis.

In this issue of the Journal, investigators from the DINO Study propose a novel coronary artery bifurcation stenosis classification system that encompasses the distribution of stenoses in the MB and/or SB, and the geometric angulation of the bifurcation..$^{13}$ The authors include the bifurcation angle in the classification because this key anatomic parameter predicts the risk of SB pinching and stenosis following provisional implantation of a stent in the main branch. ${ }^{14}$ The new DINO classification system is very similar to the widely utilized Medina classification for coronary bifurcation stenoses; ${ }^{12}$ however, instead of utilizing numbers to denote $\mathrm{MB}$ or SB involvement, DINO utilizes mnemonic letters to denote stenosis in the MB and/or SB artery (P, proximal; $\mathrm{D}$, distal; S, side branch), and incorporates the bifurcation angle ( $\mathrm{Y}<70^{\circ}$, $\mathrm{T} 70-90^{\circ}$, Reverse $>90^{\circ}$ ). Bifurcation angle was included in early classification systems ${ }^{11}$ that have largely been replaced by the Medina system which is easy to use in clinical practice. The angle of bifurcation however, certainly influences the decision to implement a provisional single vs. 2-stent treatment strategy because narrow angles $\left(<70^{\circ}\right)$ increase the risk of plaque shift into the SB and thus increase the chances of needing to perform bail-out stenting of the pinched SB. The influence of bifurcation angle on lesion complexity was con-

The opinions expressed in this article are not necessarily those of the editors or of the Japanese Circulation Society.

Received January 5, 2011; accepted January 5, 2011; released online January 19, 2011

Cardiovascular Division, Department of Medicine, Brigham and Women's Hospital, Harvard Medical School, Boston, MA, USA

Mailing address: Kevin Croce, MD, PhD, Cardiovascular Division, Brigham and Women's Hospital, Harvard Medical School, 77 Ave.

Louis Pasteur, NRB 742, Boston, MA 02115, USA. E-mail: kcroce@partners.org

ISSN-1346-9843 doi:10.1253/circj.CJ-10-1306

All rights are reserved to the Japanese Circulation Society. For permissions, please e-mail: cj@j-circ.or.jp 


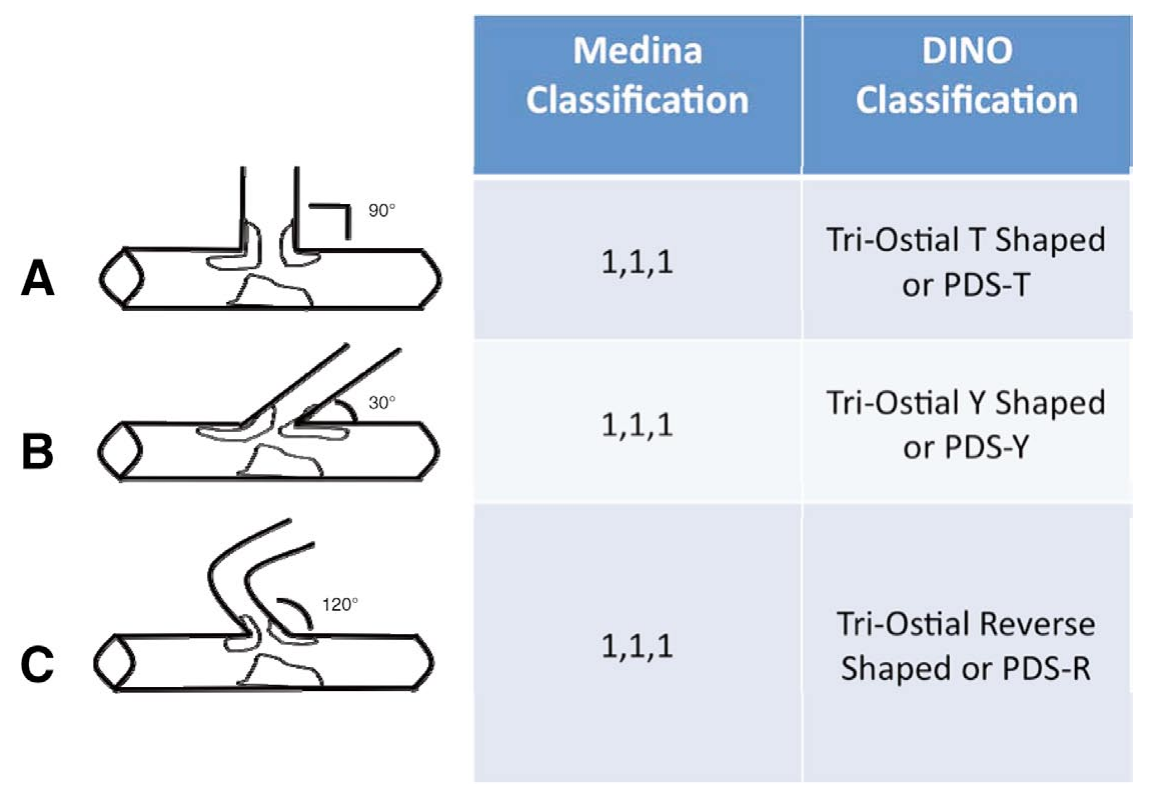

Figure. Comparison of 3 coronary bifurcation stenosis types using the Medina and DINO classification systems.

sidered in the landmark SYNTAX trial that examined the efficacy of coronary artery bypass grafting vs. multivessel PCI. In SYNTAX, an angiographic scoring system was used to characterize the complexity of PCI revascularization. In calculating the SYNTAX score, the presence of bifurcation stenoses added 1 or 2 points to the score, and an additional point was added if the angle was less than $70^{\circ} .{ }^{15}$ Bifurcation classification systems, such as DINO, can potentially be utilized to classify stenoses in an effort to predict the efficacy of a particular bifurcation stenting strategy in optimizing SB patency. Detailed bifurcation classification system might also help assess PCI complexity when trying to decide between multivessel PCI vs. coronary artery bypass revascularization. For example, a proximal and distal $90 \%$ bifurcation stenoses with a $90^{\circ}$ angle and $90 \%$ stenotic SB involvement (Medina 1,1,1; DINO Tri-ostial T-shaped or PDS-T, Figure A) might be best addressed by provisional or T-stenting, while a proximal and distal $90 \%$ bifurcation stenoses with a $30^{\circ}$ angle and $90 \%$ SB involvement (Medina 1,1,1; DINO Tri-ostial Y-shaped or PDS-Y, Figure B) might be best addressed by more complex culotte stenting. As outlined in Figure, these 2 situations would be classified identically in the Medina system, despite that fact that one would expect very different outcomes with regard to SB patency if a provisional 1-stent technique was used. When comparing the DINO classification system to the Lefreve classification system that also includes information on the bifurcation angle,${ }^{11}$ the DINO system adds further information by incorporating a new reverse shaped bifurcation category that is not addressed in the Lefreve system. The reversed shape category describes bifurcations where the MB and SB angle is greater than $90^{\circ}$. In these $>90^{\circ}$ 'reversed' bifurcations, there is elevated risk of plaque shift into the SB, and there is the potential for extreme difficulty wiring and/or passing balloons and stents into the retroflexed SB should bail-out PCI of a pinched SB be required.

As outlined above, clinical investigations have demonstrated provisional stenting of the MB is preferred, and there is no benefit to routinely employing an 'intention-to-treat' 2stent approach for the SB stenosis. Despite attempts to utilize the preferred provisional strategy, up to $30 \%$ of provisionally-treated bifurcation PCI require implantation of a second stent in a pinched or stenotic SB when the SB has a large diameter $(>2.0 \mathrm{~mm})$, supplies a large territory, or the stenosis is associated with poor flow, ischemic symptoms, or ischemic electrocardiogram changes. ${ }^{3}$ In addition, we currently have very little data on the optimal techniques for treating left main bifurcation stenoses where residual branch stenosis is less likely to be tolerated.

Bifurcation classification systems are useful clinical tools because they can potentially predict the need to convert from a provisional PCI strategy to a 2 -stent technique, and they provide a common nomenclature for research evaluating bifurcation PCI treatment strategies, devices, and outcomes. Although the DINO classification improves upon the Medina system by incorporating bifurcation angle, its use of English nomenclature (vs. numerals in the Medina system) might limit its international adoption. In addition, extensive clinical investigation will be required to determine if the DINO system predicts the need to convert from a provisional to 2-stent strategy, or predicts procedural success with a particular bifurcation stenting approach. With the anticipated development of dedicated bifurcation stents, ${ }^{3}$ classification systems that incorporate severity of $\mathrm{MB}$ and $\mathrm{SB}$ stenosis and angle might be useful in choosing a stent type, or in assessing the acute and long-term clinical outcomes for a specific bifurcation stenosis type and treatment technique/device combination.

\section{Disclosure}

Supported in part by a grant from Capes Foundation-Ministry of Education of Brazil (to A.M.), Jorge Paulo Lemann Cardiovascular Grant (to J.M.) a grant from the National Heart, Lung, and Blood Institute (1K08HL086672) (to K.C.), and a Harris Family Foundation Award (to K.C.). 


\section{References}

1. Myler RK, Shaw RE, Stertzer SH, Hecht HS, Ryan C, Rosenblum J, et al. Lesion morphology and coronary angioplasty: Current experience and analysis. J Am Coll Cardiol 1992; 19: 1641-1652.

2. Tanabe K, Hoye A, Lemos PA, Aoki J, Arampatzis CA, Saia F, et al. Restenosis rates following bifurcation stenting with sirolimuseluting stents for de novo narrowings. Am J Cardiol 2004; 94: $115-118$.

3. Latib A, Colombo A, Sangiorgi G. Bifurcation stenting: Current strategies and new devices. Heart 2009; 95: 495-504.

4. Steingen TK, Maeng M, Wiseth R, Erglis A, Kumsars I, Narbute I, et al; Nordic PCI Study Group. Randomized study on simple versus complex stenting of coronary artery bifurcation lesions: The Nordic bifurcation study. Circulation 2006; 114: 1955-1961.

5. Colombo A, Moses JW, Morice MC, Ludwig J, Holmes DR Jr, Spanos V, et al. Randomized study to evaluate sirolimus-eluting stents implanted at coronary bifurcation lesions. Circulation 2004; 109: $1244-1249$.

6. Pan M, de Lezo JS, Medina A, Romero M, Segura J, Pavlovic D, et al. Rapamycin-eluting stents for the treatment of bifurcated coronary lesions: A randomized comparison of a simple versus complex strategy. Am Heart J 2004; 148: 857-864.

7. Ferenc M, Buettner HJ, Beste Horn HP. Bifurcation Bad Krozingen. Systematic versus provisional T-stenting in the treatment of the novo coronary bifurcation lesions using sirolimus-eluting stents. Transcatheter Cardiovascular Therapeutics (TCT), October 24, 2007; Washington, DC.

8. Colombo A, Bramucci E, Saccà S, Violini R, Lettieri C, Zanini R, et al. Randomized Study of the Crush Technique Versus Provisional
Side-Branch Stenting in True Coronary Bifurcations: The CACTUS (Coronary Bifurcations: Application of the Crushing Technique Using Sirolimus-Eluting Stents) Study. Circulation 2009; 119: 71 78.

9. George BS, Myler RK, Stertzer SH, Clark DA, Cote G, Shaw RE, et al. Balloon angioplasty of coronary bifurcation lesions: The kissing balloon technique. Cathet Cardiovasc Diagn 1986; 12: $124-$ 138.

10. Pompa J, Bashore T. Qualitative and quantitative angiography bifurcation lesions. In: Topol E, editor. Textbook of interventional cardiology. Philadelphia: W.B. Saunders, 1994; 1055-1058.

11. Lefèvre T, Louvard Y, Morice MC, Dumas P, Loubeyre C, Benslimane A, et al. Stenting of bifurcation lesions: Classification, treatments and results. Cathet Cardiovasc Interv 2000; 49: $274-$ 283.

12. Medina A, Suárez de Lezo J, Pan M. A new classification of coronary bifurcation lesions. Rev Esp Cardiol 2006; 59: 183 (in Spanish).

13. Y-Hassan S, Lindroos MC, Sylvén C. A novel descriptive, intelligible and ordered (DINO) classification of coronary bifurcation lesions: Review of current classifications. Circ J 2011; 75: 299305 .

14. Godino C, Al-Lamee R, La Rosa C, Morici N, Latib A, Ielasi A, et al. Coronary left main and non-left main bifurcation angles: How are the angles modified by different bifurcation stenting techniques? J Interven Cardiol 2010; 23: 382-393.

15. Sianos G, Morel MA, Kappetein AP, Morice MC, Colombo A, Dawkins K, et al. The SYNTAX Score: An angiographic tool grading the complexity of coronary artery disease. EuroIntervention 2005; 1: 219-227. 